\title{
Review
}

\section{Kinesin superfamily proteins: roles in osteosarcoma}

\author{
Yao Lu ${ }^{1}$, Tao Song ${ }^{1}$, Xue Xue ${ }^{2}$, Gaolu Cao ${ }^{1, *}$, Panpan Huang ${ }^{1, *}$ \\ ${ }^{1}$ School of Basic Medicine, Gannan Medical University, 341000 Ganzhou, Jiangxi, China, ${ }^{2}$ Independent researcher, \\ No.93-1 Xuefu road, Jingkou district, 212001 Zhenjiang, Jiangsu, China
}

\section{TABLE OF CONTENTS}

\section{Abstract}

2. Introduction

3. Biological functions of KIFs

4. KIFs in cancer biology

5. Development of KIFs targeting inhibitors

6. KIFs in osteosarcoma

$$
\begin{aligned}
& \text { 6.1 KIFs in osteosarcoma metastasis } \\
& 6.2 \mathrm{KIFs} \text { in osteosarcoma prognosis } \\
& 6.3 \mathrm{KIFs} \text { in osteosarcoma treatment }
\end{aligned}
$$

\section{Summary and perspective}

8. Author contributions

9. Ethics approval and consent to participate

10. Acknowledgment

11. Funding

12. Conflict of interest

13. References

\section{Abstract}

Background: Osteosarcoma is a common bone tumor with extremely high malignancy, occurring mostly in children and adolescents. At present, the survival rate of osteosarcomas has made progress in some aspects; however, this can only be regarded as a partial success because substantial progress has not been made in the last few decades. Object: The kinesin superfamily is a group of proteins that play regulatory roles in various metabolic processes and are closely related to tumor metastasis. Increasing evidence shows that kinesins play key roles in the occurrence and development of human cancer. Purpose: This review summarizes the roles of the kinesin superfamily proteins in osteosarcoma and related functions.

\section{Introduction}

Osteosarcoma is one of the most common primary malignant bone tumors, with a 5-year survival of $70 \%$ to $80 \%$ [1]. It usually occurs in adolescents aged between 10 and 25, and its prevalence is slightly higher in men than in women [2]. Osteosarcoma is a rare disease but is still considered the third most common cancer owing to its high

metastasis and mortality rates [3, 4]. The focal feature of osteosarcoma is that approximately $80 \%-90 \%$ of tumors grow in the long bones of the limbs, $60 \%$ of which are located around the knees, although it may also occur in other bones such as the femur, tibia, fibula, humerus, ulna, and pelvis [5]. The malignancy of osteosarcoma is characterized by a high rate of metastases. Approximately $85 \%-90 \%$ of osteosarcomas are transferred to the lungs, $8 \%-10 \%$ are transferred to the bones, and a few are transferred to the lymph nodes [6-8]. At present, patients with osteosarcoma are treated by removing the primary lesions, removing small lung metastases, and inhibiting lung metastasis, combined with multi-drug chemotherapy using cisplatin, carboplatin, etoposide, and isocycline [9, 10]. In addition, phosphoramide, doxorubicin, and high-dose methotrexate can reduce the malignant transformation of primary tumors, but approximately $50 \%$ of osteosarcoma patients still relapse [11]. The prognosis of patients with primary osteosarcoma and lung metastases is still very poor, and the 5-year overall survival rate is only $25 \%$. Therefore, it is important to continue studies on the mechanism of osteosarcoma and discover novel treatment methods and target molecules.

The kinesin superfamily is a class of conserved microtubule-dependent molecular motor proteins with 
adenosine triphosphatase (ATPase) activity and motor properties [12]. Studies have shown that abnormal expression of the kinesin superfamily proteins (KIFs) is related to the development and progression of various human cancers [13, 14]. For example, Wang et al. [15] showed that KIF2A is highly expressed in osteosarcoma, and inhibiting the expression of KIF2A can effectively prevent the spread and migration of tumors and the invasion of osteosarcoma cells in vitro, as well as block tumor growth and metastasis in mice [16]. Gu et al. [17] showed that KIF18B is highly expressed in osteosarcoma tissues and cells and that inhibiting the expression of KIF18B significantly inhibits the proliferation, migration, and invasion of osteosarcoma cells, as well as the tumor formation ability. Other studies have shown that KIFs can be used as biomarkers for the diagnosis and treatment of osteosarcoma. This review emphasizes the importance of KIFs in the pathobiology of osteosarcoma and discusses their potential clinical uses.

\section{Biological functions of KIFs}

KIFs were first discovered in 1985. To date, 45 mouse- and human-derived KIFs have been identified, and they have been classified into 14 families (kinesin-1 to kinesin-14) according to their molecular characteristics $[18,19]$. The original kinesin, also known as KIF5/kinesin1 , is a tetramer protein consisting of two motor active heavy chains (110-120 kDa) and two light chains (60-70 kDa). All KIFs heavy chains have a highly conserved motor domain, including ATP binding sequence and microtubule binding sequence, which can bind to ATP, hydrolyse ATP, and transfer chemical energy, enabling KIFs to carry out mechanical movement along microtubules, while by combining with specific cargo, including vesicles, organelles, macromolecules, chromosomes and spindle fibres through light chains [20, 21]. Thus, the KIFs protein is involved in a variety of biological functions within the cell.

KIFs participate in a variety of biological functions in cells, including mitosis, meiosis, membrane transport, mRNA and protein transport, signal transduction, and microtubule transport [12, 22, 23]. A growing body of evidence indicates the importance of KIFs in regulating many physiological events, including brain function, developmental patterns, and even tumorigenesis [19]. Abnormal expression of KIFs plays a key role in the occurrence and development of various human cancers, by affecting the even distribution of chromosomes, leading to changes in genetic material during mitosis and the formation of abnormal spindles, thereby resulting in cytokinesis defects and mitotic arrest. Defective genetic material induces multiple functional defects in daughter cells, which may promote tumorigenesis and tumor cell invasion and metastasis $[14,24]$. Therefore, a better understanding of the function of KIFs may help to develop drugs for molecular targeted therapies against various human cancers.

\section{KIFs in cancer biology}

Abnormal expression and functions of KIFs are closely related to the development of a variety of human cancer types (Table 1, Ref. [25-64]). KIF5B, a member of the kinesin-1 family, is mainly involved in lysosomal membrane and mitochondrial transport. KIF5B is highly expressed and may be used as a diagnostic marker in neurofibromas, breast cancer [25], lung cancer [26], bladder cancer [65], skin cancer [66], and gastric cancer [67].

Table 1. Kinesin expression in cancers.

\begin{tabular}{|c|c|c|c|}
\hline Kinesins & Member (s) & $\begin{array}{c}\text { Expression in } \\
\text { cancer }\end{array}$ & Tumor type \\
\hline \multirow{3}{*}{ Kinesin-1 } & \multirow{3}{*}{ KIF5B } & \multirow{3}{*}{ High } & Neurofibromatosis [27] \\
\hline & & & Breast cancer [25] \\
\hline & & & Lung cancer [26] \\
\hline \multirow{5}{*}{ Kinesin-2 } & \multirow{5}{*}{ KIF3A, KIF3B } & \multirow{5}{*}{ High } & Lung cancer [28] \\
\hline & & & Brain tumor [29] \\
\hline & & & Breast cancer [30] \\
\hline & & & Colorectal cancer [31] \\
\hline & & & Bladder cancer [32] \\
\hline \multirow{3}{*}{ Kinesin-3 } & \multirow{3}{*}{ KIF1A, KIF1B KIF14 } & \multirow{3}{*}{ High } & Brain tumor [33] \\
\hline & & & Colon cancer [34] \\
\hline & & & Oral cancer [35] \\
\hline \multirow{5}{*}{ Kinesin-4 } & \multirow{5}{*}{ KIF4A, KIF7 } & \multirow{5}{*}{ High } & Colorectal cancer [36] \\
\hline & & & Lung cancer [37] \\
\hline & & & Gastric cancer [38] \\
\hline & & & Oral cancer [39] \\
\hline & & & Breast cancer [40] \\
\hline \multirow{5}{*}{ Kinesin-5 } & \multirow{5}{*}{ KIF11 } & \multirow{5}{*}{ High } & Breast cancer [41] \\
\hline & & & Colorectal cancer [42] \\
\hline & & & Gastric cancer [43] \\
\hline & & & Ovarian cancer [44] \\
\hline & & & Oral cancer [45] \\
\hline \multirow{5}{*}{ Kinesin-6 } & \multirow{5}{*}{ KIF20B, KIF23 } & \multirow{5}{*}{ High } & Oral cancer [46] \\
\hline & & & Breast cancer [47] \\
\hline & & & Colorectal cancer [48] \\
\hline & & & Bladder cancer [49] \\
\hline & & & Pancreatic cancer [50] \\
\hline \multirow{2}{*}{ Kinesin-7 } & \multirow{2}{*}{ KIF10 } & \multirow{2}{*}{ High } & Liver cancer [51] \\
\hline & & & Breast cancer [47] \\
\hline \multirow{5}{*}{ Kinesin-8 } & \multirow{5}{*}{ KIF18A } & \multirow{5}{*}{ High } & Prostate cancer [52] \\
\hline & & & Gastric cancer [53] \\
\hline & & & Lung cancer [54] \\
\hline & & & Breast cancer [55] \\
\hline & & & Liver cancer [56] \\
\hline Kinesin-10 & KIF22 & High & Breast cancer [57] \\
\hline \multirow{2}{*}{ Kinesin-11 } & \multirow{2}{*}{ KIF26B } & High & Breast cancer [58] \\
\hline & & III"II & Osteosarcoma [59] \\
\hline Kinesin-12 & KIF15 & High & Breast cancer [60] \\
\hline & (1) & & Osteosarcoma [61] \\
\hline & & & Breast cancer [47] \\
\hline Kinesin-13 & KIF2C & High & Gastric cancer [62] \\
\hline & & & Colorectal cancer [63] \\
\hline Kinesin-14 & KIFC1, KIFC3 & High & Breast cancer [64] \\
\hline
\end{tabular}


KIF3A and KIF3B are members of the kinesin-2 family. Kinesin-2 is a heterotrimeric complex composed of a KIF3A/3B heterodimer and KAP3. KAP3 can bind KIF3A/KIF3B to other functional proteins, such as adenomatous polyposis coli and breast tumor kinase, which are involved in regulating the occurrence of tumors [14, 68]. Kinesin-3 family proteins act as organelle transporters. There are three members in this family, namely KIF1A, $\mathrm{KIF} 1 \mathrm{~B}$, and KIF14, and these are mainly involved in the transport of mitochondria and synaptic vesicles [69, 70]. KIF1B plays a key role in nerve cell apoptosis; low expression of KIF1B can protect nerve cells and exert an antiapoptotic effect, and therefore, low expression of KIF1B is positive for the 5-year overall survival rate and metastasisfree survival rate of breast cancer patients [71].

KIF4A is a member of the kinesin-4 family and participates in the regulation of mitosis and cytokinesis of eukaryotic cells. Cells cannot complete mitosis and cytoplasmic separation in the absence of KIF14. This suggests that targeting KIF14 may be a new cancer treatment strategy. Additionally, KIF14 is a prognostic marker of breast cancer. At present, changes in the expression of KIF4A can be observed in different types of human cancers. For example, Narayan et al. [72] found that the expression of KIF4A mRNA in cervical cancer was much higher than that in normal tissues. Taniwaki et al. [37] demonstrated that inhibiting the expression of KIF4A in non-small cell lung cancer cells inhibited their growth and that patients with tumors with high KIF4A expression had a poorer prognosis than patients with tumors expressing KIF4A. KIF7 is another kinesin-4 family member. KIF7 is an effective inhibitor of the Hedgehog pathway [73] and is activated in a variety of tumors. Recent studies have shown that blocking the Hedgehog pathway may be a treatment-related cancer strategy $[74,75]$.

KIF11 is a member of the kinesin- 5 family and is overexpressed in breast, colorectal, gastric, ovarian, and oral cancers. Overexpression of KIF11 is associated with cancer staging and recurrence [76-78]. For example, in laryngeal squamous cell carcinoma, high KIF11 expression is associated with lymph node metastasis, TNM staging, and poor prognosis [79]. In oral cancer, significantly high expression of KIF11 is associated with a shorter survival time [45].

KIF20B and KIF23 are kinesin-6 family members and have been identified as potential biomarkers that promote the progression of multiple cancers [46-50]. The kinesin-7 family member KIF10 can be used as a survival and prognostic biomarker as well as a potential therapeutic target for liver [51] and breast [47] cancers. The expression of KIF18A is abnormally increased in most cancer cells. Overexpression of KIF18B is associated with poor prognosis of liver cancer [80, 81]. Furthermore, KIF18B is an oncogene of cervical cancer [82]. Heriberto et al. [57] showed that KIF22 is overexpressed in hyperplastic breast cancer tissues and can also be used as one of the biomarkers of breast cancer. KIF26B plays an important role in kidney development and is involved in the occurrence and development of certain types of tumors, including breast cancer, esophageal adenocarcinoma, and colorectal adenomatous polyposis [58, 83-85]. KIF2C is significantly upregulated in gastric and colon cancer tissues, and the 5-year overall survival rate of gastric and colon cancer patients with high expression of KIF2C is much lower than that of patients with low expression of KIF2C [62, 63]. KIFC1 overexpression in breast cancer cells increases paclitaxel resistance, and the combination of KIFC1 inhibitors with paclitaxel is a novel treatment for breast cancer [64].

\section{Development of KIFs targeting inhibitors}

Since multiple members of the KIFs have tumorigenic properties, scientists were keeping research the specific inhibitor. Eg5, the protein is encoded by the gene KIF11 which involved in the progression and development in a variety of tumor types. Monastrol as a small molecules blocks tumor cell proliferation growth by targeting specifically Eg5 and not microtubules [86]. This finding was significant. Some traditional chemo drugs based on microtubule targeting agents, such as taxol, are associated with severe side effects $[87,88]$, since all cells, healthy and tumor cells, need microtubule functions. Therefore, a variety of Eg5 inhibitors have been developed, a certain of them have entered clinical trials, including Arry-520 (Sponsor: Array Biopharma) [89-92]; LY2523355 (Sponsor: EliLilly) [93, 94]; 4SC-205 (Sponsor: 4SC); ALN-VSP02 (Sponsor: Alnylam) [95]; ispinesib (Sponsor: GSK) [96100]; AZD4877 (Sponsor: AstraZeneca) [101, 102]; SB743921 (Sponsor: Cytokinetics) [103]; ARQ 621 (Sponsor: ArQule); MK-0731 (Sponsor: Merck) [104]. Moreover, as the development of drug resistance, the strategies of reversing drug resistance were researching, and alterations in tubulin interactions are an important aspect of these strategies. Kinetin modulates docetaxel resistance by interacting with microtubules [13], and thus kinesin is assumed to be the main target of cancer chemotherapy. However, the mechanisms of PTX- and docetaxel resistance and their relationship with kinesin remain to be elucidated [64].

\section{KIFs in osteosarcoma}

As noted previously, there are 14 KIFs [12, 18] that are mainly involved in formation of the intracellular spindle in cells, chromosomal reorganization and arrangement, and cytokinesis [105]. The abnormal expression and functions of KIFs are closely related to the development of a variety of human cancers. Increasing evidence shows that KIFs may be used as molecular therapeutic targets for human cancers. Furthermore, the detection of KIFs expression in human cancers may provide biomarkers suitable for 
Table 2. Roles of kinesins in osteosarcoma.

\begin{tabular}{lcccc}
\hline Kinesins & Member (s) & Preclinical or clinical model of OS & Expression in osteosarcoma & Functions \\
\hline Kinesin-2 & KIF3B & 143B, MG-63, U2OS, SOSP9607, SJSA-1, and HOS & High & Proliferation, migration, invasion [106] \\
Kinesin-11 & KIF26B & SJSA-1, G-292, Nude Mouse Tumor Model & High & Proliferation and tumorigenesis [59] \\
Kinesin-12 & KIF15 & MNNG/HOS, U2OS, human osteosarcoma tissue & High & Proliferation, migration, invasion [61] \\
Kinesin-13 & KIF2A & MG-63 and U2OS human osteosarcoma tissue & High & Proliferation, migration, invasion, \\
& & HOS, U2OS, and Saos-2, human osteosarcoma & High & tumorigenesis [16] \\
Kinesin-8 & KIF18B & tissue, Nude Mouse Tumor Model & & Proliferation, migration, invasion, \\
& & & tumorigenesis cell cycle [17]
\end{tabular}

early detection and prognosis of human cancers [24].

At present, there are relatively few studies on KIFs in osteosarcoma. Available literature suggests that KIFs may be used as diagnostic biomarkers and targets for the treatment of osteosarcoma; however, it is necessary to further study the roles of KIFs in osteosarcoma to discover novel markers for the clinical diagnosis and identify novel targets for the prognosis and treatment of osteosarcoma. Based on the important roles of KIFs in various cancers, we have summarized the functional roles of KIFs in osteosarcoma in Table 2 (Ref. [16, 17, 59, 61, 106]) and the following sections.

\subsection{KIFs in osteosarcoma metastasis}

Osteosarcoma is a highly invasive and metastatic tumor. Current research on the functional roles of KIFs in osteosarcoma shows that some KIFs are closely related to the migration and invasion ability of osteosarcoma cells. For example, Gu et al. [106] showed that KIG3B levels are high in osteosarcoma cell lines and can promote the proliferation, migration, and invasion of osteosarcoma cells. Pu et al. [59] and others found that inhibiting the expression of KIF26B increased the sensitivity of osteosarcoma cell lines to a variety of drugs, including doxorubicin, etoposide, methotrexate, cisplatin, and carboplatin. Wu et al. [61] reported that the expression of KIF15 in osteosarcoma tissues was much higher than that in adjacent tissues. Knockdown of KTF15 in osteosarcoma cells significantly inhibited the proliferation of osteosarcoma cells and DNA synthesis in the S phase and promoted cell apoptosis. Similarly, inhibition of KTF15 expression reduced the migration and invasion of osteosarcoma cells [61].

Wang et al. [16] found that the expression of KIF2A in human osteosarcoma tissues was much higher than that in adjacent tissues, and KIF2A promoted the proliferation, migration, and invasion of osteosarcoma cells. Inhibiting the expression of KIF2A in osteosarcoma cell lines reduced their tumorigenesis and their ability to metastasize to the lung [16]. A study by Gao et al. [17] demonstrated that KIF18B is a potential oncogene and is highly expressed in osteosarcoma tissues and cells; furthermore, they demonstrated that KIF18B can promote osteosarcoma in vivo and increase osteosarcoma cell proliferation and migration in vitro.
High metastasis is a malignant manifestation of osteosarcoma. Metastatic osteosarcoma has a poor prognosis and a high recurrence rate. Therefore, the study of genes closely related to metastasis of osteosarcoma from the molecular biology perspective may facilitate the identification of targeted drugs for osteosarcoma.

\subsection{KIFs in osteosarcoma prognosis}

Because osteosarcoma is highly metastatic, the prognosis of patients with metastatic osteosarcoma is very poor, with a 5-year overall survival rate of only $25 \%$. Therefore, timely and accurate diagnosis is of great significance for effective treatment of the disease and improvement of the overall survival rate of patients. At present, the diagnosis of osteosarcoma is very limited. More than $90 \%$ of the early diagnosis of osteosarcoma in children and young/adult patients occurs based on bone pain and a non-pathological fractures. Some patients experience local swelling and a limited range of motion of the affected limb. Overall, the clinical symptoms are usually mild, lasting for several months, and are often not valued by doctors and patients [107-109]. Therefore, accurate or auxiliary diagnosis is particularly important for the early recognition of osteosarcoma.

Studies have shown that KIF3B, KIF26B, KIF15, KIF2A, and KIF18B are all highly expressed in osteosarcoma tissues and cells and can act on oncogenes to promote the proliferation and migration of osteosarcoma cells. Groth-Pedersen et al. [110] showed that interference with the expression of KIF11, KIF20A, KIF21A, and KIF25 in human osteosarcoma U2OS cells significantly reduced their proliferation. Therefore, the auxiliary detection of KIFs expression in patients with suspected osteosarcoma may assist in the diagnosis of osteosarcoma, and KIFs may be used as a new biological marker for the diagnosis of osteosarcoma.

\subsection{KIFs in osteosarcoma treatment}

Currently, the most common osteosarcoma treatment involves surgical resection of the lesion, supplemented by chemotherapy and radiotherapy. However, these treatments have certain limitations, for example, poor curative effect on patients with metastatic osteosarcoma, high recurrence rate, and poor prognosis [111]. Therefore, exploring new treatments is extremely critical for patients with metastatic osteosarcoma. Liu et al. [112] found that 


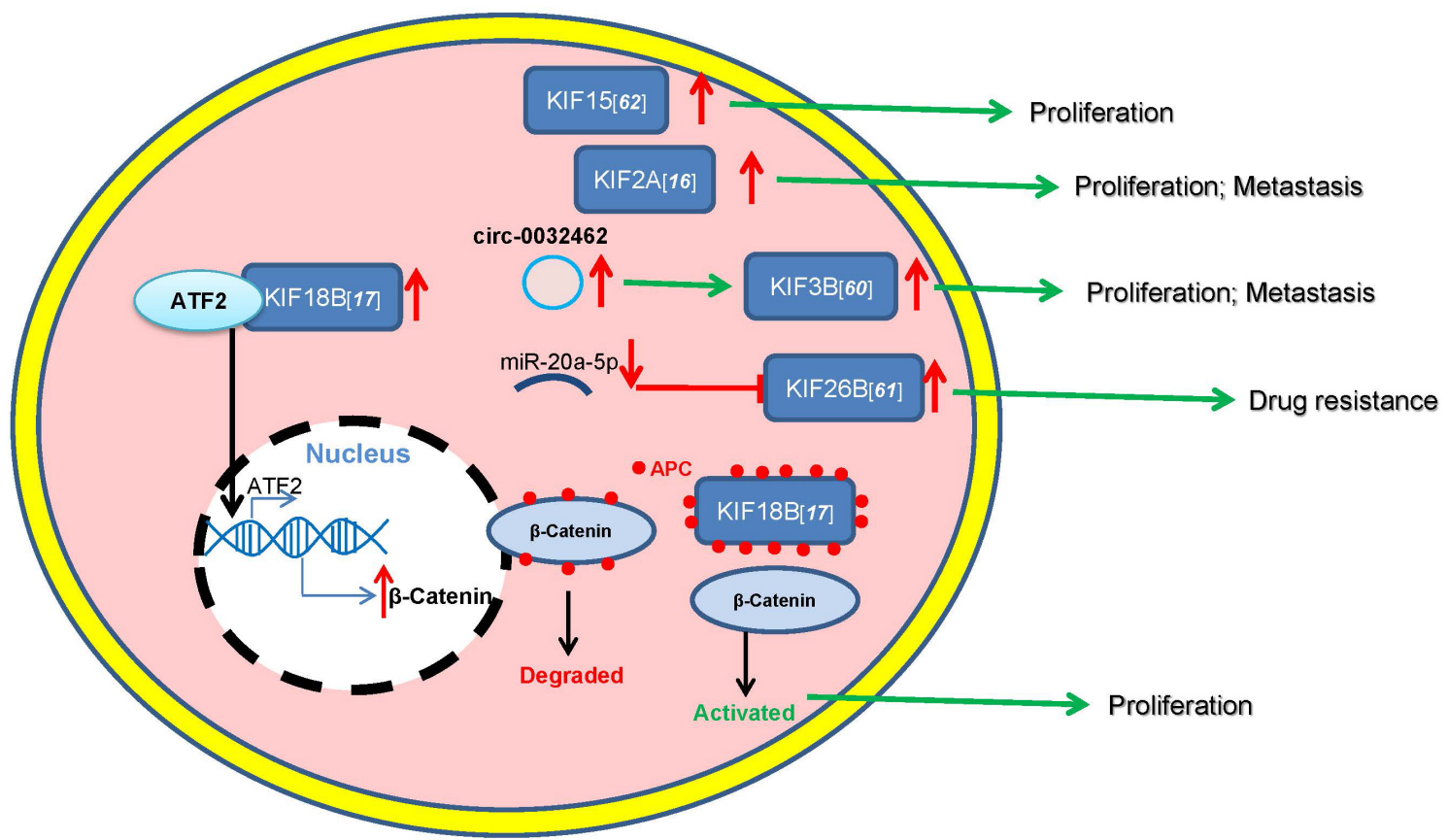

Fig. 1. Roles of KIFs in osteosarcoma.

intrathecal injection of a KIF17 antisense oligodeoxynucleotide into an osteosarcoma mouse model could relieve pain, inhibit tumor growth, and increase the survival rate after surgery. Saeki et al. [113] indicated that inhibiting the expression of KIF15 and KIF11 in osteosarcoma cell lines U2OS and HOS that are anti-S-trityl L-cysteine can significantly reduce the anti-S-trityl L-cysteine ability of these cells. Pu et al. [59] found that inhibiting the expression of KIF26B in the osteosarcoma cell line SJSA-1 reduced the resistance of these cells to multiple drugs such as doxorubicin, etoposide, cisplatin, and carboplatin. Gao et al. [17] found that inhibiting the expression of KIF18B in vivo decreased the tumor growth in U2OS osteosarcoma cells. Overall, these findings suggest that KIFs may be used as new targets for the treatment of osteosarcoma.

The identified studies indicate that KIFs participats in multiple modes of regulation for the development of osteosarcoma. (a) High-expression of KIF15 promotes osteosarcoma proliferation. (b) High-expression of KIF2A promotes osteosarcoma proliferation and metastasis. (c) CircRNA0032462 is high expression and promotes osteosarcoma proliferation and metastasis by enhancing the expression of KIF3B. (d) MiR-20a-5p can be used as a potential targeted drug to inhibit drug resistance of osteosarcoma by inhibiting the expression of KIF26B. (e) KIF18B upregulates $\beta$-catenin expression at the transcriptional level by transporting transcription factor ATF2 into the nucleus and at the post-transcriptional level by competitive binding with the adenomatous polyposis coli (APC), then releasing activated $\beta$-catenin in osteosarcoma cells. Finally, KIF18B promotes the proliferation of osteosarcoma through the above mechanisms (Fig. 1).

\section{Summary and perspective}

Osteosarcoma is the most common primary bone malignant tumor in children and adolescents, with high metastatic rates [114]. Routine surgical treatment combined with systemic chemotherapy and directed radiotherapy can increase the 5-year survival rate of patients to $70 \%$. However, the long-term survival rate of patients with metastatic or recurrent osteosarcoma is still less than $20 \%$ [115].

Abnormal KIFs expression and function are closely related to the development of a variety of human cancer types. KIFs play key roles in osteosarcoma. A variety of KIFs (KIF3B, KIF26B, KIF15, KIF2A, and KIF18B) are highly expressed in osteosarcoma tissues and cells and are closely related to the invasion and metastasis of osteosarcoma. KIF11, KIF20A, KIF21A, and KIF25 can regulate the proliferation of osteosarcoma cells, and inhibiting the expression of these KIFs can reduce the proliferative ability of osteosarcoma cells. In addition, interfering with the expression of KIF17 in the mouse sheath can relieve pain and inhibit tumor growth, as well as improve postoperative survival, whereas inhibiting the expression of KIF15, KIF11, and KIF26B can reduce the resistance of osteosarcoma cell lines to multiple drugs. Therefore, in-depth research on KIFs may provide novel markers and targets for clinical diagnosis as well as prognosis and treatment of osteosarcoma. 


\section{Author contributions}

YL, TS, XX, GLC and PPH designed this review. YL, TS and XX drafted the manuscript. GLC and PPH revised and approved the final version of the manuscript. All authors critically revised the manuscript, and read and approved the final version of the manuscript.

\section{Ethics approval and consent to participate}

Not applicable.

\section{Acknowledgment}

This work was supported by Pan-Pan Huang and Gao-Lu Cao. I thank Tao Song, Xue Xue for their critical reading of this review.

\section{Funding}

This research received no external funding.

\section{Conflict of interest}

The authors declare no conflict of interest.

\section{References}

[1] Ferrari S, Meazza C, Palmerini E, Tamburini A, Fagioli F, Cozza $\mathrm{R}$, et al. Nonmetastatic osteosarcoma of the extremity. Neoadjuvant chemotherapy with methotrexate, cisplatin, doxorubicin and ifosfamide. an Italian Sarcoma Group study (ISG/OS-Oss). Tumori Journal. 2014; 100: 612-619.

[2] Chow LTC, Wong SKC. Epiphyseal osteosarcoma revisited: four illustrative cases with unusual histopathology and literature review. Acta Pathologica, Microbiologica, et Immunologica Scandinavica. 2015; 123: 9-17.

[3] Geller DS, Gorlick R. Osteosarcoma: a review of diagnosis, management, and treatment strategies. Clinical Advances in Hematology \& Oncology. 2010; 8: 705-718.

[4] Gatta G, Botta L, Rossi S, Aareleid T, Bielska-Lasota M, Clavel J, et al. Childhood cancer survival in Europe 1999-2007: results of EUROCARE-5- a population-based study. Lancet Oncology. 2014; 15: 35-47.

[5] Jeon D, Koh JS, Cho WH, Song WS, Kong C, Cho SH, et al. Clinical outcome of low-grade central osteosarcoma and role of CDK4 and MDM2 immunohistochemistry as a diagnostic adjunct. Journal of Orthopaedic Science. 2015; 20: 529-537.

[6] Marina N, Gebhardt M, Teot L, Gorlick R. Biology and Therapeutic Advances for Pediatric Osteosarcoma. Oncologist. 2004; 9: 422-441.

[7] Chou AJ, Geller DS, Gorlick R. Therapy for Osteosarcoma: where do we go from here? Pediatric Drugs. 2008; 10: 315327.

[8] Bacci G, Longhi A, Bertoni F, Briccoli A, Versari M, Pignotti $\mathrm{E}$, et al. Bone metastases in osteosarcoma patients treated with neoadjuvant or adjuvant chemotherapy: the Rizzoli experience in 52 patients. Acta Orthopaedica. 2006; 77: 938-943.

[9] Meyers PA, Schwartz CL, Krailo M, Kleinerman ES, Betcher D, Bernstein ML, et al. Osteosarcoma: a randomized, prospective trial of the addition of ifosfamide and/or muramyl tripeptide to cisplatin, doxorubicin, and high-dose methotrexate. Journal of Clinical Oncology. 2005; 23: 2004-2011.

[10] Whelan JS, Bielack SS, Marina N, Smeland S, Jovic G, Hook $\mathrm{JM}$, et al. EURAMOS-1, an international randomised study for osteosarcoma: results from pre-randomisation treatment. Annals of Oncology. 2015; 26: 407-414.

[11] Slade AD, Warneke CL, Hughes DP, Lally PA, Lally KP, HayesJordan AA, et al. Effect of concurrent metastatic disease on survival in children and adolescents undergoing lung resection for metastatic osteosarcoma. Journal of Pediatric Surgery. 2016; 50: 157-60; discussion 160.

[12] Miki H, Okada Y, Hirokawa N. Analysis of the kinesin superfamily: insights into structure and function. Trends in Cell Biology. 2005; 15: 467-476.

[13] Tan MH, De S, Bebek G, Orloff MS, Wesolowski R, DownsKelly E, et al. Specific kinesin expression profiles associated with taxane resistance in basal-like breast cancer. Breast Cancer Research and Treatment. 2012; 131: 849-858.

[14] Yu Y, Feng Y. The role of kinesin family proteins in tumorigenesis and progression. Cancer. 2010; 116: 5150-5160.

[15] Hung P, Hong T, Hsu Y, Chen H, Chang Y, Wu C, et al. The motor protein KIF14 inhibits tumor growth and cancer metastasis in lung adenocarcinoma. PLoS ONE. 2013; 8: e61664.

[16] Wang Z, Ren S, Chang Z, Ren J. Identification of Kinesin Family Member 2a (KIF2a) as a Promising Therapeutic Target for Osteosarcoma. BioMed Research International. 2020; 2020: 7102757.

[17] Gao T, Yu L, Fang Z, Liu J, Bai C, Li S, et al. KIF18B promotes tumor progression in osteosarcoma by activating $\beta$ catenin. Cancer Biology \& Medicine. 2020; 17: 371-386.

[18] Lawrence CJ, Dawe RK, Christie KR, Cleveland DW, Dawson SC, Endow SA, et al. A standardized kinesin nomenclature. Journal of Cell Biology. 2004; 167: 19-22.

[19] Miki H, Setou M, Kaneshiro K, Hirokawa N. All kinesin superfamily protein, KIF, genes in mouse and human. Proceedings of the National Academy of Sciences. 2001; 98: 7004-7011.

[20] Okada Y, Hirokawa N. Mechanism of the single-headed processivity: diffusional anchoring between the K-loop of kinesin and the $\mathrm{C}$ terminus of tubulin. Proceedings of the National Academy of Sciences of the United States of America. 2000; 97: 640-645.

[21] Okada Y, Yamazaki H, Sekine-Aizawa Y, Hirokawa N. The neuron-specific kinesin superfamily protein KIF1a is a unique monomeric motor for anterograde axonal transport of synaptic vesicle precursors. Cell. 1995; 81: 769-780.

[22] Goldstein LS, Philp AV. The road less traveled: emerging principles of kinesin motor utilization. Annual Review of Cell and Developmental Biology. 1999; 15: 141-183.

[23] Hirokawa N, Noda Y, Tanaka Y, Niwa S. Kinesin superfamily motor proteins and intracellular transport. Nature Reviews Molecular Cell Biology. 2009; 10: 682-696.

[24] Huszar D, Theoclitou M, Skolnik J, Herbst R. Kinesin motor proteins as targets for cancer therapy. Cancer Metastasis Reviews. 2009; 28: 197-208.

[25] Moamer A, Hachim IY, Binothman N, Wang N, Lebrun J, Ali S. A role for kinesin-1 subunits KIF5B/KLC1 in regulating epithelial mesenchymal plasticity in breast tumorigenesis. EBioMedicine. 2019; 45: 92-107.

[26] Kohno T, Ichikawa H, Totoki Y, Yasuda K, Hiramoto M, Nammo $\mathrm{T}$, et al. KIF5B-RET fusions in lung adenocarcinoma. Nature Medicine. 2012; 18: 375-377.

[27] Hakimi M, Speicher DW, Shiekhattar R. The motor protein kinesin-1 links neurofibromin and merlin in a common cellular pathway of neurofibromatosis. Journal of Biological Chemistry. 2002; 277: 36909-36912.

[28] Kim M, Suh YA, Oh JH, Lee BR, Kim J, Jang SJ. KIF3A binds to $\beta$-arrestin for suppressing $\mathrm{Wnt} / \beta$-catenin signalling independently of primary cilia in lung cancer. Scientific Reports. 2016; 6: 32770. 
[29] Hoang-Minh LB, Deleyrolle LP, Siebzehnrubl D, Ugartemendia G, Futch H, Griffith B, et al. Disruption of KIF3a in patientderived glioblastoma cells: effects on ciliogenesis, hedgehog sensitivity, and tumorigenesis. Oncotarget. 2016; 7: 7029-7043.

[30] Wang W, Zhang R, Wang X, Wang N, Zhao J, Wei Z, et al. Suppression of KIF3a inhibits triple negative breast cancer growth and metastasis by repressing Rb-E2F signaling and epithelialmesenchymal transition. Cancer Science. 2020; 111: 1422 1434.

[31] Li YF, Pei FL, Cao MZ. CircRNA_101951 promotes migration and invasion of colorectal cancer cells by regulating the KIF3A-mediated EMT pathway. Experimental and Therapeutic Medicine. 2020; 19: 3355-3361.

[32] Zhou Q, Yu J, Zheng Q, Wu T, Ji Z, Zhuo Y. Kinesin family member 3A stimulates cell proliferation, migration, and invasion of bladder cancer cells in vitro and in vivo. FEBS Open Bio. 2021; 11: 1487-1496.

[33] Gan KJ, Akram A, Blasius TL, Ramser EM, Budaitis BG, Gabrych DR, et al. GSK3 $\beta$ Impairs KIF1A Transport in a Cellular Model of Alzheimer's Disease but Does Not Regulate Motor Motility at S402. eNeuro. 2020; 7: ENEURO.0176-20.2020.

[34] Hirabayashi S, Hayashi M, Nakayama G, Mii S, Hattori N, Tanabe $\mathrm{H}$, et al. The Significance of Molecular Biomarkers on Clinical Survival Outcome Differs Depending on Colon Cancer Sidedness. Anticancer Research. 2020; 40: 201-211.

[35] Pattani KM, Zhang Z, Demokan S, Glazer C, Loyo M, Goodman S, et al. Endothelin Receptor Type B Gene Promoter Hypermethylation in Salivary Rinses is Independently Associated with Risk of Oral Cavity Cancer and Premalignancy. Cancer Prevention Research. 2010; 3: 1093-1103.

[36] Matsumoto Y, Saito M, Saito K, Kanke Y, Watanabe Y, Onozawa $\mathrm{H}$, et al. Enhanced expression of KIF4a in colorectal cancer is associated with lymph node metastasis. Oncology Letters. 2018; 15: 2188-2194.

[37] Taniwaki M, Takano A, Ishikawa N, Yasui W, Inai K, Nishimura $\mathrm{H}$, et al. Activation of KIF4a as a prognostic biomarker and therapeutic target for lung cancer. Clinical Cancer Research. 2007; 13: 6624-6631.

[38] Gao J, Sai N, Wang C, Sheng X, Shao Q, Zhou C, et al. Overexpression of chromokinesin KIF4 inhibits proliferation of human gastric carcinoma cells both in vitro and in vivo. Tumour Biology. 2011; 32: 53-61.

[39] Minakawa Y, Kasamatsu A, Koike H, Higo M, Nakashima D, Kouzu Y, et al. Kinesin family member 4a: a potential predictor for progression of human oral cancer. PLoS ONE. 2013; 8: e85951.

[40] Zou JX, Duan Z, Wang J, Sokolov A, Xu J, Chen CZ, et al. Kinesin family deregulation coordinated by bromodomain protein ANCCA and histone methyltransferase MLL for breast cancer cell growth, survival, and tamoxifen resistance. Molecular Cancer Research. 2014; 12: 539-549.

[41] Zhou J, Chen W, Yang L, Wang J, Sun J, Zhang W, et al. KIF11 Functions as an Oncogene and is Associated with Poor Outcomes from Breast Cancer. Cancer Research and Treatment. 2019; 51: 1207-1221.

[42] Imai T, Oue N, Sentani K, Sakamoto N, Uraoka N, Egi H, et al. KIF11 is Required for Spheroid Formation by Oesophageal and Colorectal Cancer Cells. Anticancer Research. 2017; 37: 47-55.

[43] Imai T, Oue N, Nishioka M, Mukai S, Oshima T, Sakamoto N, et al. Overexpression of KIF11 in Gastric Cancer with Intestinal Mucin Phenotype. Pathobiology. 2017; 84: 16-24.

[44] Shi B, Bao J, Liu Y, Shi J. Death receptor 6 promotes ovarian cancer cell migration through KIF11. FEBS Open Bio. 2018; 8: 1497-1507.

[45] Daigo K, Takano A, Thang PM, Yoshitake Y, Shinohara M, Tohnai I, et al. Characterization of KIF11 as a novel prognostic biomarker and therapeutic target for oral cancer. International Journal of Oncology. 2018; 52: 155-165.

[46] Li Z, Wang Z, Li C. Kinesin family member 20B regu- lates tongue cancer progression by promoting cell proliferation. Molecular Medicine Reports. 2019; 19: 2202-2210.

[47] Li T, Zeng H, Shan Z, Ye R, Cheang T, Zhang Y, et al. Overexpression of kinesin superfamily members as prognostic biomarkers of breast cancer. Cancer Cell International. 2020; 20: 123.

[48] Lin W, Lin X, Fu S, Yang L, Tang C, Gao Y, et al. Pseudopodassociated protein KIF20B promotes Gli1-induced epithelialmesenchymal transition modulated by pseudopodial actin dynamic in human colorectal cancer. Molecular Carcinogenesis. 2018; 57: 911-925.

[49] Kanehira M, Katagiri T, Shimo A, Takata R, Shuin T, Miki T, et al. Oncogenic role of MPHOSPH1, a cancer-testis antigen specific to human bladder cancer. Cancer Research. 2007; 67: 3276-3285.

[50] Ansari D, Andersson R, Bauden MP, Andersson B, Connolly JB, Welinder $\mathrm{C}$, et al. Protein deep sequencing applied to biobank samples from patients with pancreatic cancer. Journal of Cancer Research and Clinical Oncology. 2015; 141: 369-380.

[51] Li X, Huang W, Huang W, Wei T, Zhu W, Chen G, Zhang J. Kinesin family members KIF2C/4A/10/11/14/18B/20A/23 predict poor prognosis and promote cell proliferation in hepatocellular carcinoma. American Journal of Translational Research. 2020; 12: 1614-1639.

[52] Zhang H, Shen T, Zhang Z, Li Y, Pan Z. Expression of KIF18A Is Associated with Increased Tumor Stage and Cell Proliferation in Prostate Cancer. Medical Science Monitor. 2019; 25: 64186428.

[53] Wang L, Yang S, Sun R, Lu M, Wu Y, Li Y. Expression of KIF18A in gastric cancer and its association with prognosis. Zhonghua Wei Chang Wai Ke Za Zhi. 2016; 19: 585-589. (In Chinese)

[54] Li X, Liu M, Zhang Z, Zhang L, Liang X, Sun L, et al. High kinesin family member $18 \mathrm{~A}$ expression correlates with poor prognosis in primary lung adenocarcinoma. Thoracic Cancer. 2019; 10: 1103-1110.

[55] Zhang C, Zhu C, Chen H, Li L, Guo L, Jiang W, et al. Kif18A is involved in human breast carcinogenesis. Carcinogenesis. 2010; 31: 1676-1684.

[56] Chen F, Zhong F. Kinesin Family Member 18a (KIF18a) Contributes to the Proliferation, Migration, and Invasion of Lung Adenocarcinoma Cells in Vitro and in Vivo. Disease Markers. 2019; 2019: 6383685.

[57] Bruzzoni-Giovanelli H, Fernandez P, Veiga L, Podgorniak M, Powell DJ, Candeias MM, et al. Distinct expression patterns of the E3 ligase SIAH-1 and its partner Kid/KIF22 in normal tissues and in the breast tumoral processes. Journal of Experimental \& Clinical Cancer Research. 2010; 29: 10.

[58] Wang Q, Zhao Z, Wang G, Hui Z, Wang M, Pan J, et al. High expression of KIF26B in breast cancer associates with poor prognosis. PLoS ONE. 2013; 8: e61640.

[59] Pu Y, Yi Q, Zhao F, Wang H, Cai W, Cai S. MiR-20a-5p represses multi-drug resistance in osteosarcoma by targeting the KIF26B gene. Cancer Cell International. 2016; 16: 64.

[60] Sheng J, Li C, Dong M, Jiang K. Identification by Comprehensive Bioinformatics Analysis of KIF15 as a Candidate Risk Gene for Triple-Negative Breast Cancer. Cancer Management and Research. 2020; 12: 12337-12348.

[61] Wu Z, Zhang H, Sun Z, Wang C, Chen Y, Luo P, et al. Knockdown of Kinesin Family 15 Inhibits Osteosarcoma through Suppressing Cell Proliferation and Promoting Cell Apoptosis. Chemotherapy. 2019; 64: 187-196.

[62] Nakamura Y, Tanaka F, Haraguchi N, Mimori K, Matsumoto T, Inoue $\mathrm{H}$, et al. Clinicopathological and biological significance of mitotic centromere-associated kinesin overexpression in human gastric cancer. British Journal of Cancer. 2007; 97: 543-549.

[63] Ishikawa K, Kamohara Y, Tanaka F, Haraguchi N, Mimori K, Inoue $\mathrm{H}$, et al. Mitotic centromere-associated kinesin is a novel marker for prognosis and lymph node metastasis in colorectal cancer. British Journal of Cancer. 2008; 98: 1824-1829. 
[64] De S, Cipriano R, Jackson MW, Stark GR. Overexpression of Kinesins Mediates Docetaxel Resistance in Breast Cancer Cells. Cancer Research. 2009; 69: 8035-8042.

[65] Dyrskjøt L, Kruhøffer M, Thykjaer T, Marcussen N, Jensen JL, Møller K, et al. Gene expression in the urinary bladder: a common carcinoma in situ gene expression signature exists disregarding histopathological classification. Cancer Research. 2004; 64: 4040-4048.

[66] Nindl I, Dang C, Forschner T, Kuban RJ, Meyer T, Sterry W, et al. Identification of differentially expressed genes in cutaneous squamous cell carcinoma by microarray expression profiling. Molecular Cancer. 2006; 5: 30.

[67] Hippo Y, Taniguchi H, Tsutsumi S, Machida N, Chong J, Fukayama M, et al. Global gene expression analysis of gastric cancer by oligonucleotide microarrays. Cancer Research. 2002; 62: 233-240.

[68] Haraguchi K, Hayashi T, Jimbo T, Yamamoto T, Akiyama T. Role of the kinesin-2 family protein, KIF3, during mitosis. Journal of Biological Chemistry. 2006; 281: 4094-4099.

[69] Nangaku M, Sato-Yoshitake R, Okada Y, Noda Y, Takemura R, Yamazaki H, et al. KIF1B, a novel microtubule plus end-directed monomeric motor protein for transport of mitochondria. Cell. 1994; 79: 1209-1220.

[70] Matsushita M, Tanaka S, Nakamura N, Inoue H, Kanazawa H. A novel kinesin-like protein, KIF1Bbeta3 is involved in the movement of lysosomes to the cell periphery in non-neuronal cells. Traffic. 2004; 5: 140-151.

[71] Pan H, Zhao X, Yuan H, Zhang W, Li X, Wang G, et al. Decreased serum IL-22 levels in patients with systemic lupus erythematosus. Clinica Chimica Acta. 2009; 401: 179-180.

[72] Narayan G, Bourdon V, Chaganti S, Arias-Pulido H, Nandula $\mathrm{SV}$, Rao PH, et al. Gene dosage alterations revealed by cDNA microarray analysis in cervical cancer: identification of candidate amplified and overexpressed genes. Genes, Chromosomes \& Cancer. 2007; 46: 373-384.

[73] Cheung HO, Zhang X, Ribeiro A, Mo R, Makino S, Puviindran $\mathrm{V}$, et al. The Kinesin Protein Kif7 is a Critical Regulator of Gli Transcription Factors in Mammalian Hedgehog Signaling. Science Signaling. 2009; 2: ra29.

[74] Tremblay MR, Lescarbeau A, Grogan MJ, Tan E, Lin G, Austad BC, et al. Discovery of a Potent and Orally Active Hedgehog Pathway Antagonist (IPI-926). Journal of Medicinal Chemistry. 2009; 52: 4400-4418.

[75] Sarangi A, Valadez JG, Rush S, Abel TW, Thompson RC, Cooper MK. Targeted inhibition of the Hedgehog pathway in established malignant glioma xenografts enhances survival. Oncogene. 2009; 28: 3468-3476.

[76] Wissing MD, De Morrée ES, Dezentjé VO, Buijs JT, De Krijger RR, Smit VTHBM, et al. Nuclear Eg5 (kinesin spindle protein) expression predicts docetaxel response and prostate cancer aggressiveness. Oncotarget. 2014; 5: 7357-7367.

[77] Sun D, Lu J, Ding K, Bi D, Niu Z, Cao Q, et al. The expression of Eg5 predicts a poor outcome for patients with renal cell carcinoma. Medical Oncology. 2013; 30: 476.

[78] Liu L, Liu X, Mare M, Dumont AS, Zhang H, Yan D, et al. Overexpression of Eg5 correlates with high grade astrocytic neoplasm. Journal of Neuro-Oncology. 2016; 126: 77-80.

[79] Lu M, Zhu H, Wang X, Zhang D, Xiong L, Xu L, et al. The prognostic role of Eg5 expression in laryngeal squamous cell carcinoma. Pathology. 2016; 48: 214-218.

[80] Itzel T, Scholz P, Maass T, Krupp M, Marquardt JU, Strand S, et al. Translating bioinformatics in oncology: guilt-by-profiling analysis and identification of KIF18B and CDCA3 as novel driver genes in carcinogenesis. Bioinformatics. 2015; 31: 216224.

[81] Shichijo S, Ito M, Azuma K, Komatsu N, Maeda Y, Ishihara Y, et al. A unique gene having homology with the kinesin family member 18a encodes a tumour-associated antigen recognised by cytotoxic T lymphocytes from HLA-a2+ colon cancer patients European Journal of Cance. 2005; 41: 1323-1330.

[82] Wu Y, Wang A, Zhu B, Huang J, Lu E, Xu H, et al. KIF18B promotes tumor progression through activating the $\mathrm{Wnt} / \beta$-catenin pathway in cervical cancer. OncoTargets and Therapy. 2018; 11 : 1707-1720.

[83] Uchiyama Y, Sakaguchi M, Terabayashi T, Inenaga T, Inoue S, Kobayashi C, et al. Kif26b, a kinesin family gene, regulates adhesion of the embryonic kidney mesenchyme. Proceedings of the National Academy of Sciences. 2010; 107: 9240-9245.

[84] Gu J, Ajani JA, Hawk ET, Ye Y, Lee JH, Bhutani MS, et al. Genome-wide catalogue of chromosomal aberrations in barrett's esophagus and esophageal adenocarcinoma: a high-density single nucleotide polymorphism array analysis. Cancer Prevention Research. 2010; 3: 1176-1186.

[85] Horpaopan S, Spier I, Zink AM, Altmüller J, Holzapfel S, Laner A, et al. Genome-wide CNV analysis in 221 unrelated patients and targeted high-throughput sequencing reveal novel causative candidate genes for colorectal adenomatous polyposis. International Journal of Cancer. 2015; 136: E578-E589.

[86] Mayer TU, Kapoor TM, Haggarty SJ, King RW, Schreiber SL, Mitchison TJ. Small molecule inhibitor of mitotic spindle bipolarity identified in a phenotype-based screen. Science. 1999 286: 971-974.

[87] Von Hoff DD. The taxoids: same roots, different drugs. Seminars in Oncology. 1997; 24: S13-S10.

[88] Takahashi M, Ohtani S, Nagai SE, Takashima S, Yamaguchi M, Tsuneizumi M, et al. The efficacy and safety of pertuzumab plus trastuzumab and docetaxel as a first-line therapy in Japanese patients with inoperable or recurrent her2-positive breast cancer: the COMACHI study. Breast Cancer Research and Treatment. 2021; 185: 125-134.

[89] Shah JJ, Kaufman JL, Zonder JA, Cohen AD, Bensinger WI, Hilder BW, et al. A Phase 1 and 2 study of Filanesib alone and in combination with low-dose dexamethasone in relapsed/refractory multiple myeloma. Cancer. 2017; 123: 4617_ 4630.

[90] Chari A, Htut M, Zonder JA, Fay JW, Jakubowiak AJ, Levy JB, et al. A phase 1 dose-escalation study of filanesib plus bortezomib and dexamethasone in patients with recurrent/refractory multiple myeloma. Cancer. 2016; 122: 3327-3335.

[91] Lee HC, Shah JJ, Feng L, Manasanch EE, Lu R, Morphey A, et al. A phase 1 study of filanesib, carfilzomib, and dexamethasone in patients with relapsed and/or refractory multiple myeloma. Blood Cancer Journal. 2019; 9: 80.

[92] Ocio EM, Motlló C, Rodríguez-Otero P, Martínez-López J, Cejalvo MJ, Martín-Sánchez J, et al. Filanesib in combination with pomalidomide and dexamethasone in refractory MM patients: safety and efficacy, and association with alpha 1-acid glycoprotein (AAG) levels. Phase Ib/II Pomdefil clinical trial conducted by the Spanish MM group. British Journal of Haematology. 2021; 192: 522-530.

[93] Infante JR, Patnaik A, Verschraegen CF, Olszanski AJ, Shaheen M, Burris HA, et al. Two Phase 1 dose-escalation studies exploring multiple regimens of litronesib (LY2523355), an Eg5 inhibitor, in patients with advanced cancer. Cancer Chemotherapy and Pharmacology. 2017; 79: 315-326.

[94] Wakui H, Yamamoto N, Kitazono S, Mizugaki H, Nakamichi S, Fujiwara Y, et al. A phase 1 and dose-finding study of LY2523355 (litronesib), an Eg5 inhibitor, in Japanese patients with advanced solid tumors. Cancer Chemotherapy and Pharmacology. 2014; 74: 15-23.

[95] Tabernero J, Shapiro GI, LoRusso PM, Cervantes A, Schwartz GK, Weiss GJ, et al. First-in-humans trial of an RNA interference therapeutic targeting VEGF and KSP in cancer patients with liver involvement. Cancer Discovery. 2013; 3: 406-417.

[96] Bradley DA, Hussain M. Promising novel cytotoxic agents and combinations in metastatic prostate cancer. Cancer Journal. 2008; 14: 15-19. 
[97] Beer TM, Goldman B, Synold TW, Ryan CW, Vasist LS, Van Veldhuizen PJ, et al. Southwest Oncology Group phase II study of ispinesib in androgen-independent prostate cancer previously treated with taxanes. Clinical Genitourinary Cancer. 2008; 6: 103-109.

[98] Knox JJ, Gill S, Synold TW, Biagi JJ, Major P, Feld R, et al. A phase II and pharmacokinetic study of SB-715992, in patients with metastatic hepatocellular carcinoma: a study of the National Cancer Institute of Canada Clinical Trials Group (NCIC CTG IND.168). Investigational New Drugs. 2008; 26: 265-272.

[99] Lee CW, Bélanger K, Rao SC, Petrella TM, Tozer RG, Wood L, et al. A phase II study of ispinesib (SB-715992) in patients with metastatic or recurrent malignant melanoma: a National Cancer Institute of Canada Clinical Trials Group trial. Investigational New Drugs. 2008; 26: 249-255.

[100] Tang PA, Siu LL, Chen EX, Hotte SJ, Chia S, Schwarz JK, et al. Phase II study of ispinesib in recurrent or metastatic squamous cell carcinoma of the head and neck. Investigational New Drugs. 2008; 26: 257-264.

[101] Gerecitano JF, Stephenson JJ, Lewis NL, Osmukhina A, Li J, Wu K, et al. A Phase i trial of the kinesin spindle protein (Eg5) inhibitor AZD4877 in patients with solid and lymphoid malignancies. Investigational New Drugs. 2013; 31: 355-362.

[102] Jones R, Vuky J, Elliott T, Mead G, Arranz JA, Chester J, et al. Phase II study to assess the efficacy, safety and tolerability of the mitotic spindle kinesin inhibitor AZD4877 in patients with recurrent advanced urothelial cancer. Investigational New Drugs. 2013; 31: 1001-1007.

[103] O’Connor OA, Gerecitano J, Van Deventer H, Hainsworth J, Zullo KM, Saikali K, et al. The addition of granulocyte-colony stimulating factor shifts the dose limiting toxicity and markedly increases the maximum tolerated dose and activity of the kinesin spindle protein inhibitor SB-743921 in patients with relapsed or refractory lymphoma: results of an international, multicenter phase I/II study. Leukemia \& Lymphoma. 2016; 56: 2585-2591.

[104] Holen K, DiPaola R, Liu G, Tan AR, Wilding G, Hsu K, et al. A phase i trial of MK-0731, a kinesin spindle protein (KSP) inhibitor, in patients with solid tumors. Investigational New Drugs. 2012; 30: 1088-1095.

[105] Zhu C, Zhao J, Bibikova M, Leverson JD, Bossy-Wetzel E, Fan $\mathrm{J}$, et al. Functional analysis of human microtubule-based motor proteins, the kinesins and dyneins, in mitosis/cytokinesis using RNA interference. Molecular Biology of the Cell. 2005; 16: 3187-3199.

[106] Gu R, Li X, Yan X, Feng Z, Hu A. Circular RNA circ_0032462 Enhances Osteosarcoma Cell Progression by Promoting KIF3B Expression. Technology in Cancer Research \& Treatment. 2020; 19: 1533033820943217

[107] Bielack SS, Kempf-Bielack B, Delling G, Exner GU, Flege $\mathrm{S}$, Helmke K, et al. Prognostic factors in high-grade osteosarcoma of the extremities or trunk: an analysis of 1,702 patients treated on neoadjuvant cooperative osteosarcoma study group protocols. Journal of Clinical Oncology. 2002; 20: 776-790.
[108] Klein MJ, Siegal GP. Osteosarcoma: anatomic and histologic variants. American Journal of Clinical Pathology. 2006; 125: $555-581$.

[109] Gianferante DM, Mirabello L, Savage SA. Germline and somatic genetics of osteosarcoma - connecting aetiology, biology and therapy. Nature Reviews Endocrinology. 2017; 13: 480491.

[110] Groth-Pedersen L, Aits S, Corcelle-Termeau E, Petersen NHT, Nylandsted J, Jäättelä M. Identification of cytoskeletonassociated proteins essential for lysosomal stability and survival of human cancer cells. PLoS ONE. 2013; 7: e45381.

[111] Kempf-Bielack B, Bielack SS, Jürgens H, Branscheid D, Berdel WE, Exner GU, et al. Osteosarcoma relapse after combined modality therapy: an analysis of unselected patients in the Cooperative Osteosarcoma Study Group (COSS). Journal of Clinical Oncology. 2005; 23: 559-568.

[112] Liu M, Liu Y, Hou B, Bu D, Shi L, Gu X, et al. Kinesin superfamily protein 17 contributes to the development of bone cancer pain by participating in NR2B transport in the spinal cord of mice. Oncology Reports. 2015; 33: 1365-1371.

[113] Saeki E, Yasuhira S, Shibazaki M, Tada H, Doita M, Masuda T, et al. Involvement of C-terminal truncation mutation of kinesin5 in resistance to kinesin-5 inhibitor. PLoS ONE. 2018; 13 e0209296.

[114] Mirabello L, Troisi RJ, Savage SA. Osteosarcoma incidence and survival rates from 1973 to 2004. Cancer. 2009; 115: 15311543.

[115] Sakamoto A, Iwamoto Y. Current status and perspectives regarding the treatment of osteo-sarcoma: chemotherapy. Reviews on Recent Clinical Trials. 2008; 3: 228-231.

Abbreviations: ATPase, adenosine triphosphatase; KIFs, kinesin superfamily protein.

Keywords: Kinesin superfamily; Osteosarcoma; Metastasis; Prognosis; Treatment

\section{Send correspondence to:}

Gaolu Cao, School of Basic Medicine, Gannan Medical University, 341000 Ganzhou, Jiangxi, China, Email: gzcgl@163.com

Panpan Huang, School of Basic Medicine, Gannan Medical University, 341000 Ganzhou, Jiangxi, China, Email: huangpp@gmu.edu.cn 